\title{
Editorials
}

\section{Divergent differentiation in soft tissue sarcomas}

Fortunately for the surgical pathologist, most soft tissue sarcomas attempt to form only one type of mesenchymal tissue, thereby facilitating diagnosis and classification. Even malignant fibrous histiocytoma-like pleomorphic sarcomas usually display a single line of differentiation when any is detectable. Additional mesenchymal elements, however, are sometimes seen in soft tissue tumours derived from neuroectoderm or mesoderm. Sarcomas which display divergent differentiation with at least two components (not patterns) apart from "fibrosarcoma" are known as malignant mesenchymomas. Stout's aim in advocating this term was to recognise the complexity of the composition of such tumours while avoiding "a host of hyphenated names" specifying all their different elements. ${ }^{12}$ The current WHO classification retains Stout's definition, but advises somewhat uneasily that the diagnosis of malignant mesenchymoma be used sparingly and only when other designations do not apply accurately. ${ }^{3}$ A further recommendation is that, notwithstanding Stout's intention, the term be qualified by the type of elements present, although certain soft tissue entities with rhabdomyosarcomatous or other lines are generally excluded. ${ }^{34}$ Among these are malignant peripheral nerve sheath tumours (which are of neuroectodermal origin) with heterologous differentiation; and "dedifferentiated" tumours, such as liposarcoma, chondrosarcoma, or chordoma, with or without heterologous elements in their otherwise undifferentiated portions.

Thus defined, malignant mesenchymomas are rare, with only eight cases in 22 years at one major referral centre. ${ }^{4}$ They form large tumours in adults, mainly in the retroperitoneum and thigh. Most are liposarcomatous and the further elements are osteochondroid, or more rarely, myoid (leiomyo- or rhabdomyosarcomatous). An example of the latter mentioned by Stout was a tumour of the leg, with gross but no histological illustration, which was composed in part of lipoblasts and cross-striated rhabdomyoblasts. In two recent series of malignant mesenchymomas, rhabdomyoblasts have been reported with well differentiated or round cell liposarcoma in neoplasms in retroperitoneum or thigh that also had osteochondroid differentiation, ${ }^{4}$ and with pleomorphic liposarcoma. ${ }^{5}$ In this issue of the Journal, a further example in the thigh is described by Shanks et $a l^{t}$ as an apparently myxoid liposarcoma, with areas of pleomorphic liposarcoma in which there were ultrastructurally confirmed rhabdomyoblasts. The adipose component of this tumour is noteworthy in passing in that the poorly differentiated variant of myxoid liposarcoma (which is well defined by its $\mathrm{t}(12 ; 16)$ (q13;p11) abnormality and resultant fusion of CHOP and TLS (FUS) genes) is round cell liposarcoma, and pleomorphic areas are not expected.

Malignant mesenchymomas have one predominant histological component; the additional ones are frequently discrete or multifocal. The definition is not quantitative, but each of the two or more tissues should be sufficiently differentiated to permit its clear recognition by accepted criteria. This case report emphasises that, in a pleomorphic sarcoma with lipoblasts, the presence of rhabdomyoblasts is easily overlooked, as can be other patterns of rhabdomyosarcoma which have been described in malignant mesenchymoma. ${ }^{5}$ Skeletal muscle differentiation can also be under-diagnosed using immunohistochemistry because the most commonly used muscle markers (except the inconstant myoglobin) are not wholly specific or sensitive; pleomorphic rhabdomyosarcoma, sometimes morphologically indistinguishable from pleomorphic leiomyosarcoma, can be desmin negative and $\alpha$-smooth muscle actin positive, ${ }^{7}$ so that other diagnostic reference points are needed. The gold standard has been provided by electron microscopy, as even rudimentary sarcomere formation provides specific evidence of skeletal muscle differentiation, but this is likely to be supplemented by the use of antibodies to $\alpha$-sarcomeric actin and to myogenic regulatory proteins, especially myogenin and $M y o \mathrm{D} 1,{ }^{8}$ once their specificity has been thoroughly established.

The clinical significance, if any, of the presence or number of rhabdomyoblasts is unclear. No quantitative study exists and, more relevantly, most reports give no information about which component has metastasised, although rhabdomyosarcoma is not always present in the recurrent tumour. ${ }^{5}$ Some patients with malignant mesenchymoma seem to do relatively well, ${ }^{5}$ but many have a generally poor prognosis on adequate follow up, ${ }^{4}$ and there are no specific treatment options beyond those common to all pleomorphic high grade sarcomas.

Diagnostic considerations might, however, generate therapeutic dilemmas when choices lie between two or more different chemotherapeutic regimens, as in bi- or polyphenotypic round cell tumours of childhood. The constituents of these can be morphologically distinct, like in the rare ectomesenchymoma, where rhabdomyosarcoma occurs in combination with neural cells of varying maturity, but in other cases the evidence for dual differentiation is primarily immunohistological, as in intraabdominal desmoplastic small round cell tumours, or revealed only at subcellular and molecular levels. For example, in a number of neoplasms histologically resembling alveolar rhabdomyosarcoma, with rhabdomyoblasts and expressing myogenin, myf5, desmin and muscle specific actin, chimeric $E W S / F L I 1$ transcripts and fusion proteins identical with those of peripheral primitive neuroectodermal tumours have also been identified. This duality was reflected ultrastructurally by the presence in the same cell of primitive neurites and dense core neurosecretory granules as well as cytoplasmic filament patterns characteristic of primitive rhabdomyosarcoma. ${ }^{9}$

Unexpected lines of differentiation continue to be recognised in various soft tissue tumours. A recent example, ${ }^{10}$ which extends and is supported by earlier ultrastructural studies, ${ }^{11}$ is the observation that epithelioid sarcoma, in which the dominant lineage is epithelial, can express the endothelial markers vascular cadherin, CD31 and CD34. As new reagents are produced and the sensitivity of our diagnostic techniques increases, further examples with more than one phenotype will be identified. For both adult and paediatric soft tissue sarcomas it will become necessary to decide the level of diagnosis at which identifi- 
cation of each component becomes clinically significant, and to investigate which of the phenotypes should determine management.

Consultant Histopathologist,

Department of Histopathology,

Royal Marsden NHS Trust,

Fulham Road,

London SW3 $67 f$

1 Stout AP. Tumors of the soft tissues. In: Atlas of tumor pathology (sect 2, fasc 5). Washington DC: Armed Forces Institute of Pathology, 1953:118-20.

Nash A, Stout AP. Malignant mesenchymomas in children. Cancer 1961;14:524-33.

3 Weiss SW. Histological typing of soft tissue tumours. WHO International Histological Classification of Tumours. 2nd edn. Berlin: Springer Verlag, 1994:46.
4 Brady MS, Perino G, Tallini G, Russo P, Woodruff JM. Malignant mesenchymoma. Cancer 1996;77:467-73.

5 Newman PL, Fletcher CDM. Malignant mesenchymoma. Clinicopathologic analysis of a series with evidence of low-grade behaviour. Am $\mathcal{F}$ Surg Pathol 1991;15:607-14.

B. Focal rhabdomyosarcomatous differentiation in primary liporcm $\mathcal{F}$ Clin Pathol 1996;49:770-772.

7 Schürch W, Bégin LR, Seemayer TA, Lagacé R, Boivin JC, Lamoureux C, et al. Pleomorphic soft tissue myogenic sarcomas of adulthood. A reappraisal $T$ in the mid 1990s. Am F Surg Pathol 1996;20:131-47.

8 Tallini GPD, Dias P, Cordon-Cardo C, Houghton PJ, Rosai J. Myogenic regulatory protein expression in adult soft tissue sarcomas. A sensitive and $\underline{-}$ specific marker of skeletal muscle differentiation. Am f Pathol 1994; 144:693-701.

9 Sorensen PH, Shimada H, Liu XF, Lim JF, Thomas G, Triche TJ. Biphenotypic sarcomas with myogenic and neural differentiation express the and Smith MEF, Brown JI, Fisher C. Evidence of endothelial differentiation in Smith MEF, Brown JI, Fisher C. Evidence of endothelial d

11 Fisher C. Epithelioid sarcoma: the spectrum of ultrastructural differentiation in seven immunohistochemically defined cases. Hum Pathol 1988;19:265-75.

\section{The coroner's necropsy - an epidemiological treasure trove}

The coroner's necropsy is suited to classic epidemiological studies, not only for those conditions leading to death but also for coincident disease. In the general population there is an iceberg of hidden pathology. This pathology may occur as pre-symptomatic, quiescent or burnt out disease. These silent processes do not necessarily appear in returns generated from hospitals or other clinical sources. The changing pattern of this disease can provide clues to causation as well as early signs that shifts in health care provision may be required.

As the coroner's investigation is a local enquiry the information is of direct relevance to the community from which it is generated. Clustering of pathology according to micro-geography of a city might give aetiological clues. For example, is there a relation with local industry? Or with sources of pollution? Or with ethnic or other community subsets? Furthermore, locally produced and available statistics encourages early and responsive health care planning, which is of benefit to the entire community.

While population epidemiology has been primarily concerned with determinants of health status of large populations, clinical epidemiology tends to deal with diagnostic and therapeutic aspects in subpopulations of diseased individuals. ${ }^{1}$ The coroner's necropsy also has a role in this area.

Sensitivity and specificity are characteristics which have been used to appraise a test. Together they assess the ability to pick up a target condition, or they can evaluate a new test according to a gold standard. These characteristics may be applied to a clinical diagnosis and the 'gold standard' necropsy result. ${ }^{2}$ For a population of patients dying in hospital, such measures indicate clinical diagnostic accuracy. It must be conceded that the reason many of these cases are referred to the coroner is that the cause of death is unknown. Even so, there will be a provisional working diagnosis or diagnoses. Thus, calculation of sensitivity and specificity for a variety of pre-mortem clinical diagnoses might form the basis for a comparison of performance between hospitals.

Care must be taken with the use of information generated from coroners' necropsies. Firstly, there will be bias in selection of deaths which are referred to the coroner and which of these are then subsequently the subject of a postmortem examination. There are clearly social pressures exerted by relatives on their doctors to avoid such examinations. Where a death has been reported but is not associated with suspicious circumstances, gentle persua- $\frac{\mathbb{D}}{\mathbb{P}}$ sion by the coroner's office may lead to issue of a death $\stackrel{\mathbb{D}}{3}$ certificate without necropsy. The medical attendants themselves may wish to avoid the spectre of an inquest. Calder has shown that $75 \%$ of the mortality due to hip fracture was under certified. ${ }^{3}$

Secondly, the yield of necropsy data and its accuracy is the subject of concern. Postmortem examinations are not yet open to rigorous audit, the exception being forō accredited forensic pathologists. At least for examinations of medical interest, clinico-pathological discussion with clinicians results in a form of peer review. No such review $\overrightarrow{\overrightarrow{0}}$ takes place with most coroners' cases.

O'Sullivan, in this issue, documents the substantial amount of information present in coroners' necropsies. ${ }^{4}$ It is unknown how much of this is being missed because of substandard examinations. The national enquiry into perioperative deaths for $1992-93$ rates $28 \%$ of all necropsy examinations as poor or not acceptable. ${ }^{5}$ The enquiry also noted that the majority of cases lacked a histology report; in $46 \%$ this resulted in a significant detraction in diagnos-?

tic value of the postmortem examination.
Information is also lost as a result of inadequacies of recording. Proposals have been made to modify certificates $N$ to include extra information. ${ }^{6}$ Since 1985 , however, any N child dying within the first 28 days of life is issued with aN certificate which takes the following form:

Cause of Death

a) Main diseases or conditions in infant.

b) Other diseases or conditions in infant.

c) Main maternal diseases or conditions affecting the infant.

d) Other maternal disease or conditions affecting infant.

e) Other relevant causes.

A certificate along similar lines could be issued aftero completion of a necropsy instead of the traditional medical certificate of cause of death. It is sometimes argued thato waiting for necropsy results delays unacceptably the issue of a death certificate, but in practice, more often than not the certificate is completed after the necropsy results are known.

The way forward for necropsy based epidemiological studies is perhaps to target a condition worthy of attention and channel efforts into generating high quality necropsy 